\title{
EQUACIONS DIFERENCIALS AMB RETARD
}

C. Perel16

L'evolucio de les equacions diferencials funcionals ês un exemple de com, encera avui, es fa matematica "pura" a partir de necessitats practiques En aquest cas, ha sigut l'entendre i poder controlar processos dinamics on apareix un retras en el temps, el que ha donat origen $i$ ha fet creixer aquesta bran ca de les matematiques.

Aquestes equacions van ser tractades, en un començament, cada una com un cas particular (per exemple per vito volterra, a principis de segle) $i$ no és sino a partir de Krasovskii, en el seu estudi de la estabilitat del moviment fet després de la segona guerra, que adquireix una estructura matenatica més coherent $i$ formal. Es en els anys cinquantes $i$ seixantes que s'es tructura tota una teoria i son Shimanov, Elsgolts, Halanay i Hale, entre d'altres, els que s'encarreguen de fer-ho. A partir d'aquells moments, la teoria s'utilitza per l'estudi de siste mes amb "memoria" (es a dir, en que el comportament depen de la seva "historia"). Avui hi ha molta gent treballant en aques tes qüestions ja molt desiligats de les motivacions originals, per estructurar dins de la matenatica aquests noug estudis o be nomes perque és un camp en el que encara es poden escriure articles sense massa dificultats, i aconseguir d'aquesta manera prestigi i feina.

Les publicacions sobre el tema són força abundants com es despren de veure la seccio corresponent al "Mathematical re views" o al "Zentralblatt".

Passarem a exposar diferents aspectes de la teoria de les 
equacions diferencials amb retard al temps.

Una equacio diferencial oroinaria relaciona els valors de les derivades d'una funcio per cada valor de la variable independent (temps), el mateix per la funcio $i$ les seves derivades.

Per exemple

$$
F\left(t, x(t), x^{\prime}(t), \ldots, x^{(n)}(t)\right)=0 .
$$

El resolare l'equacio és trobar $x$ que la satisfagui.

En els sistemes dinamics reals, pero. ens trobem algunes vegades en que hi ha retards temporals. Per exemple en el control de la velocitat de rotaci6 d una turbina de vapor o del flux de neutrons dun reactor nuclear, hi ha temps finits de transmissió de senyals.

Les equacions que descriven aquests sistemes relacionen les derivades (la taxa temporal de la variacio de l'estat) amb els valors de la funcio (estat) en temps anteriors.

$L$ 'equacis més senzilla que se m'acut que encara tingui algún interes il. lustratiu es

$$
x^{\prime}(t)=x(t-2)
$$

El problema de trobar solucions d'aquesta equacio és ben diferent de trobar solucions per

$$
x^{+}(t)=x(t) \text {. }
$$

que té per anica solucio amb valor $\xi$ per $t=0$ la funcio $x(t)=g e^{t}$.

Si per solucio de la primera equacio entenem tota funcio definida en un cert interval, que sigui continua, tingui deri vada per la oreta $i$ satisfagui l'equacio, tenim que $n$ 'hi ha moltes de solucions $x$ definides $a[0, \infty)$ tals que $x(0)=\xi$. 
Una $a^{\prime}$ elles s'obte prenent $x(t)=\xi e^{\lambda t}$ amb $\lambda=e^{-\lambda}$. Una altra és la que val $g$ a I'interval $[-1,0]$, i que per tota $t$ a $[0, \infty)$ val

$$
x([t])+\int_{[t]}^{t} x(\tau-1) d \tau \text {. }
$$

amb $[t]:=$ part entera de $t$.

La mateixa formula dona una solucio de la equacio a $\mathbb{R}^{+}$ si es defineix $x(t)=\varphi(t)$ per $t \varepsilon[-1,0]$, sent $\varphi$ qualsevol funcis continua a $[-1,0]$.

Notem que en general no existeixen solucions que siguin extensions d'aquestes: que estiguin definides per valors de $t$ menors que -1 .

Resulta d'aquest exemple que, $3 i$ volem un problema "ben plantejat", hem de considerar els estats no corn a punts, és a dir, els valors de $x(t)$, sino con a funcions definides en un interval, que en el nostre cas és de longitud $l$.

prenent com el nostre espai a'estats l'espai $c([-1,0], \mathbb{R}) \quad($ abreu. $c)$, de les funcions reals continues a linterval $[-1.0]$, amb la metrica

$$
d(\varphi, \Psi)=\sup \{|\varphi(\tau)-\Psi(t)|: r[-1,0]\} .
$$

tenim que per cada $\varphi \in C$ existeix una anica solucio $x$ tal que $x(\tau)=\varphi(\tau)$ per $\tau \varepsilon[-1,0]$. Si ara denotem per $x_{t} I^{\prime}$ element de C definit per $x_{t}(\tau)=x(t+\tau)$ per $\tau \in\{-1,0\}, i$ amb $x_{t}(\varphi)$ fen notar la dependencia en el valox inicial $\varphi$. resulta que $x_{t}(\varphi)$ representa una trajectoria a $C$, és a dir, una aplicacio continua de $[0, \infty)$ a $c$.

Notem que és valida la propietat $x_{s+t}(\varphi)=x_{s}\left(x_{t}(\varphi)\right)$, el que ens diu que definint $T(t) \varphi=x_{t}(\varphi), T(t): C \rightarrow C$ per $t \geq 0$ 
es un semigrup de transformacions de $\mathrm{C}$ amb $\mathrm{T}(0)=I$, la iden titat.

Resulta que $T(t) \varphi$ és continua en $\varphi$ i encara ltés, que si definim l'aplicacio

$$
\begin{aligned}
\mathrm{T}: c \times \mathbb{R}^{+} & \rightarrow \mathrm{c} \\
(\varphi, t) & \rightarrow \mathrm{x}_{t}(\varphi)=\mathrm{T}(t)_{\varphi .} .
\end{aligned}
$$

aquesta es continua i compleix

$$
\begin{aligned}
& T(t)\left(T(s)_{\varphi}\right)=T(t+s)_{\varphi} \\
& T(0)=I .
\end{aligned}
$$

Tota aplicacio $T$ anb aquestes propietats es coneguda com a "semisistema dinamic". A diferència del cas dels sistemes ä namics (definits per equacions diferencials ordinaries, per exemple), en que $\mathbb{R}^{+}$és sustituit per $\mathbb{R}$, ara no es $T(t, \cdot)$ necessariament un homeonorfigme $i(T(t))^{-1}$ pot no existir. De fet, resulta del teorema de Arzela que $\mathrm{T}(\mathrm{t})$ és compacte per $t \geq I$, el que vol dir que envia la bola unitaria de $c$ (que no é compacta), en un conjunt compacte.

Tot aquest comportament es generalitza a d'altres equacions. Consideren les successives generalitzacions:

0)

$$
\begin{aligned}
& \dot{x}^{*}(t)=x(t-1) \\
& x^{*}(t)=F\left(x_{t}\right)
\end{aligned}
$$

en que $F: C(\{-r, 0], \mathbb{R}) \rightarrow \mathbb{R}$ es lineal i contínua

- ir es un real positiu (per comptes de

1 que hem fet servir abans)

2)

$$
x^{*}(t)=E\left(x_{t}\right),
$$

on $x$ pren valors a $\mathbb{R}^{n} i$

$F: C\left([-r, 0], \mathbb{R}^{n}\right) \rightarrow \mathbb{R}^{n}$ és Iineal i contínua

$$
x^{\prime}(t)=F\left(x_{t}\right)
$$


en que $F$ no és necessariament lineal i es localment lipschitziana.

En aquest cas podria no existir la solucio $x_{t}(\varphi)$ per tota $t \in \mathbb{R}^{+}$, pero $s i F(\varphi)$ es $O(\varphi)$, si que existeix $i$ queda definit igualment un semisistema dinamic a $C:=c\left([-r, 0], \mathbb{R}^{n}\right)$.

$$
x^{*}(t)=F\left(t, x_{t}\right) \text {. }
$$

en que $\mathrm{F}$ es contínua i localment lipschitziana respecte a la segona variable.

En aquest cas no es té definit un semisistema dinamic a $C$, gind a $C \times \mathbb{R}$ prenent $t^{\prime}=1$.

Podria ser que es definís un semisistema dinànic amb altres tipus d'equacions, per exemple. $x^{*}(t)=F\left(x(t-1), x^{\prime}(t-1)\right)$. on a $F$ hi entren també les derivades de $x$. Aquesta darrera equacio és del tipus "neutre" i les que hem esmentat abans son del tipus "retardat".

De moment nomes parlarem del comportament de les més senzilles, les del tipus 2) diguem:

$$
x^{\prime}(t)=F\left(x_{t}\right)
$$

amb $F$ lineal $i$ continua.

En el cas analeg d'equacions diferencials ordinaries $x^{\prime}(t)=F(x(t))=A x(t)$, el que es fa és canviar coordenades de manera que $A$ quedi en forma candnica de Jordan. Resulta que cada bloc de Jordan dóna un subespai invariant $i$ que $\mathbb{R}^{n}$ es suma directa d'aquests.

A cada un d'aquests subespais el comportament fs ben conegut:

$$
x(t)=\xi e^{A t}, \text { on, al ser }
$$




$$
A=\left(\begin{array}{cccc}
\lambda & 1 & \ldots & 0 \\
\vdots & & & \vdots \\
0 & \ldots & 1 \\
1 & \cdots & \lambda
\end{array}\right)
$$

es te

$$
e^{A t}=e^{\lambda t}\left(\begin{array}{ccc}
1 & \frac{t^{n-1}}{(n-1) !} \\
\vdots & \vdots \\
0 & \cdots & 1
\end{array}\right)
$$

En el nostre cas ens agradaria poguer fer quelcom de semblant, $i$ el que es pot fer és descomposar $C$ en suma direç ta d'un subespai invariant de dimensi6 finita, on el comportament de les solucions escomel de les de $x^{\prime}=A x$ (amb $A$ en for ma candnica), i un subespai de dimensio infinita er que les solucions tendeixen a 0 , tant mes rapidament com mes gran prenem la dimensio d'A.

De fet podem expressar les solucions com a combinacio (infinita) de solucions a subsespais del tipus esmentat, modul Euncions que tendeixen a zero més depressa que qualsevol expo Hencial.

Per fer-ho s'ha de repetir el que es fa en equacions diferencials ordinaries en una bona direccio.

Hem de partir, a l'equacio $x^{\prime}=A x$, no de $A$ sino de les solucions $x(t, \xi)=\xi e^{A t}=: T(t) \cdot \xi$.

Aixo és un grup uniparametric de transformacions de $\mathbb{R}^{\mathrm{n}}$ en $\mathbb{R}^{n}$. És fortament continu a $\mathbb{R}, T(t)$ és continu per cada $t$, $\lim _{T \rightarrow t}|T(t) \xi-T(T) g|=0$ per tota $t i$ el seu generador in Einitessimal, que per cada $x$ és $\lim _{t \rightarrow 0} \frac{1}{t}(T(t) x-x)$, es precisament $A$. 
A les equacions com a 2) fem el mateix. Tenim el mateix semigrup $T(t)$ definit com al principi per les solucions, $i$ el seu generador infinitessimal A donat per

$$
A_{\varphi}=\lim _{t \rightarrow 0^{+}} \frac{1}{t}(T(t) \phi-\phi) .
$$

Aquest operador es tancat $i$ té per domini, no tot $C$, sino un subespai dens donat per les funcions en que $\varphi(0)=F(\varphi)$. En aquest subespai es te

$$
A_{\varphi}(\tau)=\varphi^{\prime}(t), \quad T \in[-r, 0]
$$

(Per les propietats dels semigrups de transformacions recomanem el llibre de Hille i Phillips.)

El funcional $F$ admet, d'acord amb la representacio de Riesz, la forma

$$
F(\varphi)=\int_{-r}^{0}\left[d_{\eta}(T)\right]_{\varphi}(t) \text {, en que } \eta \text { és una funcio }
$$

(matricial de $n \times n$ ) de variacio acotada.

Resulta que A té un espectre purament puntual que ve donat per les arrels de

$$
\Delta(\lambda):=\operatorname{det}\left(\lambda I-\int_{-r}^{0} e^{\lambda \tau} d_{\eta}(\tau)\right)=0 .
$$

Si s'estudien aquestes arrels (complexes). es veu que totes tenen part real mes petita que cert valor $i$ que, entre dos valors qualsevols, no $n$ 'hi ha més que un nombre finit que hi tinguin la part real compresa.

Si $\lambda$ esta a $l$ 'espectre $\sigma(A)$ de $A$ i prenem $U_{k} N(\langle A-\lambda I) \mathbf{k}): M_{\lambda}$ resulta que $M_{h}$ esta contingut al domini de $A$. es invariant sota $A$ i tambe sota $T(t): T(t) A=A T(t)$ a $D(A)$. I no tan sols 
aixo, sino que cada $M_{\lambda}$ es de dimensib finita $d_{\lambda}$.

Si prenem un conjunt finit de valors propis $A=\left\{\lambda_{1}, \ldots, \lambda_{p}\right\} \quad\left(\lambda_{t} \in \sigma(A)\right)$, resulta que

$$
C=M_{\lambda_{1}}+\cdots \oplus M_{\lambda_{D}} \oplus Q .
$$

A cada $M_{k}=: M$ poden escollir una base $=\left(\varphi_{1}, \ldots \varphi_{d}\right)$, pre sa com a matriu $n \times d$. Si $\mathrm{B}$ és la matriu d $\times$ d definida per $A \Phi=\Phi$ es té que $I$ 'unic valor canctentic de $B$ és $\lambda$, $i$ es pot fer, escollint be $B$, que tingui forma candnica de Jordan. Resuita llavors, que si $\phi=$ G $\in M, T(t) \varphi=e^{B t} a_{;} \cdot \ldots$ $t \geq 0 .\left(\Phi(\tau)=\Phi(0) e^{B_{\tau}}\right)$.

Aquesta expressio tambe te sentit i satisfa l'equacio per $t<0$. Per tant a les $m_{\alpha}$ les solucions son definides per tota $t \varepsilon \mathbb{R}$, i son com les de $I^{\prime}$ equacio $x^{\prime}=B x$.

Ara be, $Q$ tambe es invariant sota $T(t) i$, el que resulta més interessant es que, si $\sup \operatorname{Re}\{0(A) \backslash A\}<\gamma$, llavors per a 0 .

$$
|T(\tau) \varphi| \leq x e^{Y t}|\varphi| \text {. }
$$

i gi y és prou petit aixd tenđeix a zero molt depressa.

Anb aixo teriin dones ja una bona imatge $i$, en particular permet esbrinar l'estabilitat de la solucio nula. Si Re $\sigma(A)<0$ es té que és asimptoticament estable $i$ si és $\leq 0$, sera estable si els blocs de Jordan dels valors propis amb part real nula son diagonals.

El trobar $\$$ no és facil, en general. Veiem-ne un exemple. I ' equacio

$$
x^{\prime \prime \prime}(t)+a x^{\prime \prime}(t)+b x^{\prime}(t)+k x(t-r)=0
$$

te per espectre d'A les arrels de

$$
\lambda^{3}+a \lambda^{2}+b \lambda+k e^{-r \lambda}=0 .
$$


El calcul d'aquestes arrels diu que tindrem dues arrels simples imaginaxies $i$ les demés amb part real negativa per certs valors dels parametres $a, b$ i $k$.

I'equacio com a sistema queda

$$
x^{\prime}(t)=A x(t)+B x(t-r) .
$$

amb

$$
A=\left(\begin{array}{ccc}
0 & 1 & 0 \\
0 & 0 & 1 \\
0 & -b & a
\end{array}\right), \quad B=\left(\begin{array}{ccc}
0 & 0 & 0 \\
0 & 0 & 0 \\
-k & 0 & 0
\end{array}\right)
$$

i

$$
\eta(\tau)=\left(\begin{array}{ccc}
0 & u(\tau) & 0 \\
0 & 0 & u(\tau) \\
-k u(\tau+r) & -b u(\tau) & -a u(\tau)
\end{array}\right),
$$

on $u(t)$ es zero per $t$ negativa i un per $t$ positiva.

$S i \pm i_{\omega}$ son les arrels imaginaries, tenim

$$
\Phi=\left(\begin{array}{ll}
e^{j \omega \tau} \tau & e^{-i \omega \tau} \\
i \omega e^{i \omega \tau} & -i \omega e^{-i \omega \tau} \\
-\omega^{2} e^{i \omega \tau} & -\omega^{2} e^{-i \omega \tau}
\end{array}\right)
$$

- bé, considerant tan sols la part real

$$
\Phi=\left(\begin{array}{cc}
\cos \omega \tau & \sin \omega \tau \\
-\omega \sin \omega \tau & \omega \cos \omega \tau \\
-\omega^{2} \cos \omega \tau & -\omega^{2} \sin \omega \tau
\end{array}\right)
$$

i. $\quad B=\left(\begin{array}{rr}0 & w \\ -\omega & 0\end{array}\right)$.

Tenim doncs que a $c$ hi ha un subespi invariant de dimen- 
sis dos, en que les solucions son con lee d'un oscil.lador harmonic (periodiques totes del mateix periode $\frac{2 \pi}{\omega}$ ), i totes les demes solucions tendeixen exponencialment a aquestes. Havent vist doncs el comportament de les solucions de 2), podern preguntar-nos ara que passa si sthi introdueix un terme depenent de $t$ :

$$
x^{\prime}(t)=F\left(\mathbf{x}_{t}\right)+f(t)
$$

La formula de variacio de parametres del halanay en dona la solucio sota' Ia forma

$$
x_{t}=T(t) \varphi+\int_{0}^{t} T(t-s) x_{0} f(s) d s
$$

on $T(t) x_{0}$ es $I a$ solucio de $l$ equacio 2) que té per condicio inicial la matriu que val 0 a $[-r, 0)$ i $I$ a 0 .

Utilitiant aquesta formula ts facil provar que per una equacio no lineal

$$
x^{*}(t)=F\left(x_{t}\right)=L\left(x_{t}\right)+N\left(x_{t}\right)
$$

en que suposem $F(0)=0$ il la derivada de fréchet de $F$. Si no hi ha valors imaginaris a lespectre del generador infini. tessimal corresponent a la part lineal, resulta que, en un en torn de 0 , hi ha varietats invariants en que les trajectories es comporten com en el cas lineal. De fet, les projeccions sobre $M$ i $Q$ son homeomorfismes i les cotes exponencials segueixen valent:

Q, per $t \geq 0 i$

$$
|T(t) \varphi| S \mathrm{Ke}^{\gamma t}|\varphi| \text { a la varietat que es projects a }
$$

$$
|T(t) \varphi| \leq K e^{\gamma^{t}}|\varphi| \text { a la varietat que es projecta }
$$

$M$, per $t \leq 0$.

Utilitzant la formula de variacio de parametres es pot ge 
neralitzar també el metode de bifurcacio, per esbrinar I'exis tència de solucions periodiques amb equacions quasi lineals (amb no linealitat afectada d'un parametre petit).

Per una altra banda, les equacions del tipus "neutre" tam be poden definir semisistemes dinamics. En particular les de la forma $\frac{d}{d t} g\left(x_{t}\right)=f\left(x_{t}\right)$.

Per exemple

$$
\frac{d}{d t}(x(t)-x(t-1))=0 \text { n'és un cas. }
$$

Notem que pot molt be ser que una solucio d'aquesta equaci6 no sigui derivable. El que si que ho es es $x(t)-x(t-1)$.

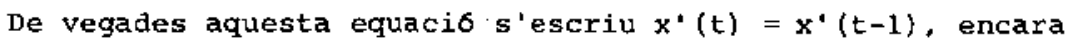
que pot no tenir sentit en certs punts.

Perque quedi definit un semisistema dinanic $s^{\text {than de res }}$ tringir $f$ i $g$ convenientment De fet, en el cas lineal, es pot demanar que $g\left(x_{t}\right)=x(t) * M\left(x_{t}\right)$ amb la funcio de variacio acotada $\mu$ que representa al funcional lineal $M$, que sigui continua a 0 .

Per poguer tenir una descomposicio en subespais invariants demanern també que $\mu$ no tingui part singular, Ess a dir., que

$$
M(\varphi)=-\int_{-r}^{0}\left(q_{1}\right) \omega=\sum_{k=1}^{\infty} a_{k} \varphi\left(-r_{k}\right)+\int_{-r}^{0} a \text {. }
$$

on $a, a_{k}$ son matrius, $0<r_{k} \leq r, a \in L_{1}[-r, 0] \underset{j}{i} \sum_{k=1}^{\infty} a_{k}$ es absolutament convergent.

La teoria per aquestes equacions és analoga a la que hem fet per 2), excepte que hi ha complicacions amb o(A) perque $T(t)$ no té perque ser compacte per cap valor de $t$. 
Els interessats poden, per obtenir més informacio i bibliografia, consultar el lijb. de J. Hale: "Functional Diffe rential Equations", publicat per Springer-Verlag, i pel cas neutre la tesi de l'autor a la Universitat Autonoma de Barce lona. 